\title{
Systematic Review \\ Weekly Semaglutide vs. Liraglutide Efficacy Profile: A Network Meta-Analysis
}

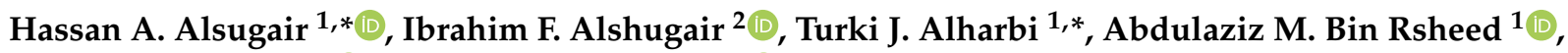 \\ Ayla M. Tourkmani ${ }^{1}$ (D) and Wedad Al-Madani ${ }^{3}$ (D) \\ 1 Family Medicine Department, Prince Sultan Military Medical City, Riyadh 12624, Saudi Arabia; \\ abdulaziz_binrsheed@yahoo.com (A.M.B.R.); aylatourkmani@gmail.com (A.M.T.) \\ 2 Orthopedic Surgery Department, King Saud Medical City, Riyadh 12746, Saudi Arabia; ishugair@gmail.com \\ 3 General Authority of Statistics, Riyadh 11481, Saudi Arabia; madaniwh@gmail.com \\ * Correspondence: alsugair.hassan@gmail.com or Halsugair@psmmc.med.sa (H.A.A.); Turkifcm@yahoo.com \\ or tjalharbi@psmmc.med.sa (T.J.A.); Tel.: +966-114777714-40438 (T.J.A.)
}

Citation: Alsugair, H.A.;

Alshugair, I.F.; Alharbi, T.J.;

Bin Rsheed, A.M.; Tourkmani, A.M.;

Al-Madani, W. Weekly Semaglutide

vs. Liraglutide Efficacy Profile: A

Network Meta-Analysis. Healthcare

2021, 9, 1125. https://doi.org/

$10.3390 /$ healthcare 9091125

Academic Editor: Francesco Sessa

Received: 25 June 2021

Accepted: 22 August 2021

Published: 30 August 2021

Publisher's Note: MDPI stays neutral with regard to jurisdictional claims in published maps and institutional affiliations.

Copyright: (c) 2021 by the authors. Licensee MDPI, Basel, Switzerland. This article is an open access article distributed under the terms and conditions of the Creative Commons Attribution (CC BY) license (https:// creativecommons.org/licenses/by/ $4.0 /)$.

\begin{abstract}
Introduction: Glucagon-like peptide 1 receptor agonist (GLP-1 RA) is a class of hypoglycemic medications. Semaglutide once-weekly (QW) and liraglutide once-daily (OD) significantly improved glycemic control compared to placebo. To date, no long-term phase III trials directly comparing semaglutide and liraglutide are available. This network meta-analysis (NMA) aims to compare the long-term efficacy of semaglutide and liraglutide. Methods: PubMed, Embase, and Cochrane Library were searched from inception until June 2019 to identify relevant articles. Nine long-term randomized controlled trials comparing once-weekly semaglutide or liraglutide with placebo or other active comparisons were identified. The outcomes of interest were changes in $\mathrm{HbA} 1 \mathrm{c}$ and weight after 52 weeks. A Bayesian framework and NMA were used for data synthesis. This is a sub-study of the protocol registered in PROSPERO (number CRD42018091598). Results: The data showed significant superiority in $\mathrm{HbA} 1 \mathrm{c}$ reduction of semaglutide $1 \mathrm{mg}$ QW over liraglutide 1.2 and $1.8 \mathrm{mg}$ with a treatment difference of $0.47 \%$ and $0.3 \%$, respectively. Semaglutide $0.5 \mathrm{mg}$ QW was found to be significantly superior to liraglutide $1.2 \mathrm{mg}$ in $\mathrm{HbA} 1 \mathrm{c}$ reduction with a treatment difference of $0.17 \%$. Regarding weight reduction analysis, semaglutide 0.5 and $1 \mathrm{mg}$ QW were significantly associated with a greater reduction than liraglutide $0.6 \mathrm{mg}$ with a treatment difference of 2.42 and $3.06 \mathrm{~kg}$, respectively. However, no significant reduction was found in comparison to liraglutide 1.2 and $1.8 \mathrm{mg}$. Conclusions: Semaglutide improved the control of blood glucose and body weight. The capacity of long-term glycemic control and body weight control of semaglutide appears to be more effective than other GLP-1 RAs, including liraglutide. However, considering the number of included studies and potential limitations, more large-scale, head-to-head, well-designed randomized-controlled trials (RCTs) are needed to confirm these findings.
\end{abstract}

Keywords: network meta-analysis; diabetes mellitus; glycemic control; HbA1c; weight; semaglutide; liraglutide; Glucagon-like peptide; GLP-1; GLP-1 RA

\section{Introduction}

Type 2 diabetes mellitus (T2DM) is a progressive and complex metabolic disorder characterized by chronic hyperglycemia due to insulin resistance and pancreatic beta cell dysfunction [1]. Chronic hyperglycemia is associated with multiple complications, including retinopathy, nephropathy, and neuropathy, in addition to various manifestations of atherosclerotic cardiovascular disease (CVD) [1,2]. According to one large randomized controlled trial (RCT) involving 11,140 participants, with every 1\% increase in glycosylated hemoglobin $(\mathrm{HbA} 1 \mathrm{c})$, risk was increased up to $40 \%$ of all-cause and cardiovascular mortality [3]. Glycemic control becomes progressively difficult with time, and advancements in therapy are needed to maintain glycemic targets $[4,5]$. 
Glucagon-like peptide 1 receptor agonist (GLP-1 RA) is a class of hypoglycemic medications that has shown benefit in glucose metabolism, beta-cell function enhancement, and weight loss promotion with a low risk of hypoglycemia [6]. GLP-1 RAs are classified into short- and long-acting preparations based on their mode of action [7,8]. Compared with other hypoglycemic medications, RCTs have reported promising long-term effects, especially with respect to cardiovascular outcomes [6]. The U.S. Food and Drug Administration (FDA) has approved several GLP-1 RAs, including exenatide twice-daily (Bid), lixisenatide once-daily (OD), liraglutide OD, exenatide once-weekly (QW), albiglutide QW, dulaglutide QW, and semaglutide QW [9-13].

The US FDA approved liraglutide in 2010 as a daily subcutaneous injection with therapeutic doses of $1.2 \mathrm{mg}$ and $1.8 \mathrm{mg}$ for T2DM $[6,10,14]$. In head-to-head RCTs, liraglutide showed a greater reduction in mean $\mathrm{HbA1c}$ and fasting plasma glucose (FPG) than exenatide Bid, exenatide QW, albiglutide $\mathrm{QW}$ and lixisenatide and was non-inferior to dulaglutide QW $[15,16]$. Additionally, RCTs reported greater weight reduction from baseline in liraglutide compared to lixisenatide, exenatide $\mathrm{QW}$, dulaglutide $\mathrm{QW}$, and albiglutide QW [16]. RCTs showed similar weight reduction in exenatide Bid compared to liraglutide [16]. The U.S. FDA approved semaglutide in 2017 as a once-weekly subcutaneous injection [9]. A 30-week phase III RCT on semaglutide reported a significant reduction in $\mathrm{HbA1c}$ and weight from baseline compared with placebo [17]. In a head-to-head comparison with dulaglutide and exenatide $\mathrm{QW}$, semaglutide was superior in achieving glycemic control and weight reduction $[18,19]$. A review published by Courtney et al. on GLP-1 medications showed clinically significant glycemic control and weight reduction of liraglutide [20]. In two recent network meta-analyses on GLP-1 RA RCTs, semaglutide was found superior to liraglutide in glycemic control and weight reduction. However, conclusions were drawn from only data gathered at $24 \pm 4$ weeks [21,22].

To date, no long-term phase III trials directly comparing semaglutide QW and liraglutide OD are available. In the absence of long-term head-to-head RCTs, network meta-analysis is a statistical method that allows the estimation of the comparative effectiveness of multiple treatments [23,24]. This network meta-analysis (NMA) compared the long-term efficacy in $\mathrm{HbA1c}$ reduction and weight change between semaglutide $\mathrm{QW}$ and liraglutide OD.

\section{Materials and Methods}

This is a sub-study of the protocol registered in PROSPERO (number CRD42018091598). This network meta-analysis was conducted in accordance with the Preferred Reporting Items for Systematic Reviews and Meta-Analyses (PRISMA) statement [25].

Database Search: Electronic database search included the PubMed, Embase, and Cochrane's Library from inception to June 2019. The key term "Liraglutide OR NN2211 OR Semaglutide OR NN9535" was used for all of the databases. The search was restricted to English, French, and Spanish language publications. The earliest publication found using the above search key-term dated back to 2001. However, early publications did not meet the inclusion criteria for this NMA.

Study Selection: This review included double-blind, single-blind, or open-label RCTs with available data on $\mathrm{HbA1c}$ or weight. In which once-weekly semaglutide ( $0.5 \mathrm{mg}$ or $1.0 \mathrm{mg}$ ) and liraglutide $(1.2 \mathrm{mg}$ or $1.8 \mathrm{mg}$ ) compared with other active intervention or placebo. RCTs with adults aged at least 18 years with T2DM and a duration of $\geq 52$ weeks on intervention were only included. Final end-point data were used for the analysis of RCTs longer than 52 weeks. Nonrandomized, experimental studies, crossover trials, and reviews in addition to studies with less than fifty participants were excluded. The eligibility of included studies was assessed independently by two reviewers (A.H.A. and A.I.F.). Discrepancies were resolved by a third reviewer (A.T.J.).

Data Extraction and Quality Evaluation: Unified extraction forms were used to extract the following data: (1) authors' information; (2) publication year; (3) demographic data including age, gender, diabetes duration, and background therapy; (4) baseline of outcome 
measures; (5) sample sizes; (6) interventions of each arm; (7) dosages of each arm; (8) outcomes of interest (see above); and (9) duration. For extension trials, data were extracted from the extension phase. Data that were not reported in the original manuscripts were retrieved from ClinicalTrials.gov (accessed on 22 August 2021). Two investigators (A.H.A. and A.I.F.) extracted data independently. The quality of eligible studies was evaluated according to the Cochrane Collaboration's risk of bias tool for assessing risk of bias [26].

Data synthesis and analysis: The network meta-analysis was conducted based on the Cochrane institute instructions to compare the efficacy of weekly semaglutide $0.5 \mathrm{mg}$ and $1 \mathrm{mg}$ versus liraglutide $1.2 \mathrm{mg}$ and $1.8 \mathrm{mg}$ for the reduction of $\mathrm{HbA} 1 \mathrm{c}$ and weight as a primary intervention [27]. Other medications went on the equation to complete the network of comparisons and they were; oral semaglutide $14 \mathrm{mg}$, liraglutide $3 \mathrm{mg}$, sitagliptin $100 \mathrm{mg}$, glimepiride $4 \mathrm{mg}$, glimepiride $8 \mathrm{mg}$, oral anti-diabetic drugs, exenatide $2 \mathrm{mg}$, and placebo. All continuous outcomes were performed using normal likelihood analysis and random effect was used for a better fit between trials. The NMA model was implemented using GeMTC software [28]. It used the Bayesian evidence network, which all indirect comparisons are taken into account to arrive at a single, integrated, estimate of the effect of all included treatments based on the included studies. The Bayesian analysis also allows assessing the consistency of the results to draw conclusions. Bayesian analysis is a type of analysis that is widely used to improve the estimate of the standard error used in a good old-fashioned $t$-test. The Bayesian has many advantages over the frequentist analysis; of them, it gives more coherent results that can be analyzed and interpreted in such a complicated review as network meta-analysis. Additionally, Bayesian inference allows for the flexible implementation of relatively complicated statistical techniques, such as those that involve hierarchical nonlinear model [29]. The results of the NMA was presented in mean treatment difference and 95\% credible intervals ( $\mathrm{Crl}$ ) for mean treatment effect. The treatment that results in a greater treatment reduction from baseline was favored. Forest plots were generated using DistillerSR [30]. Surface under the cumulative ranking curve (SUCRA) was used for ranking interventions for each outcome [31].

\section{Results}

The search process yielded 12,315 results. Of these, 11,492 results were manually excluded during initial screening, as we did not augment the search filter to exclude publications by language, animal vs. human design, published protocols, reviews, and so forth. By abstract screening, 629 results were excluded. This was followed by a fulltext assessment. Of these, 101 were excluded as they did not meet the inclusion criteria. Furthermore, 85 results were excluded due to duplication or not reporting the outcomes of interest. As a result, nine studies were included in the final review. Flowchart of trial selection is shown in Figure 1.

\subsection{Study Characteristics}

Interventions included in the analysis once-weekly semaglutide $0.5 \mathrm{mg}$, once-weekly semaglutide $1 \mathrm{mg}$, once-daily liraglutide $1.2 \mathrm{mg}$, once-daily liraglutide $1.8 \mathrm{mg}$, once-daily oral semaglutide $14 \mathrm{mg}$, once-daily liraglutide $3 \mathrm{mg}$, once-weekly exenatide ER $2 \mathrm{mg}$, oncedaily sitagliptin $100 \mathrm{mg}$, once-daily glimepiride $4 \mathrm{mg}$, once-daily glimepiride $8 \mathrm{mg}$, and metformin 1500-2000 mg daily. Overall, nine trials with a total of 9618 patients included to the analysis. The publication year ranged from 2009 to 2018. Trial duration ranged from 52 to 104 weeks. Trials design and baseline characteristics are shown in Tables 1 and 2, respectively. Overall, the risk of bias was low in the included studies. However, due to limited long-term studies on liraglutide, $60 \%$ completion rate was accepted. Additionally, other biases that were due to the interference of the pharmaceutical companies in funding studies were permitted. 


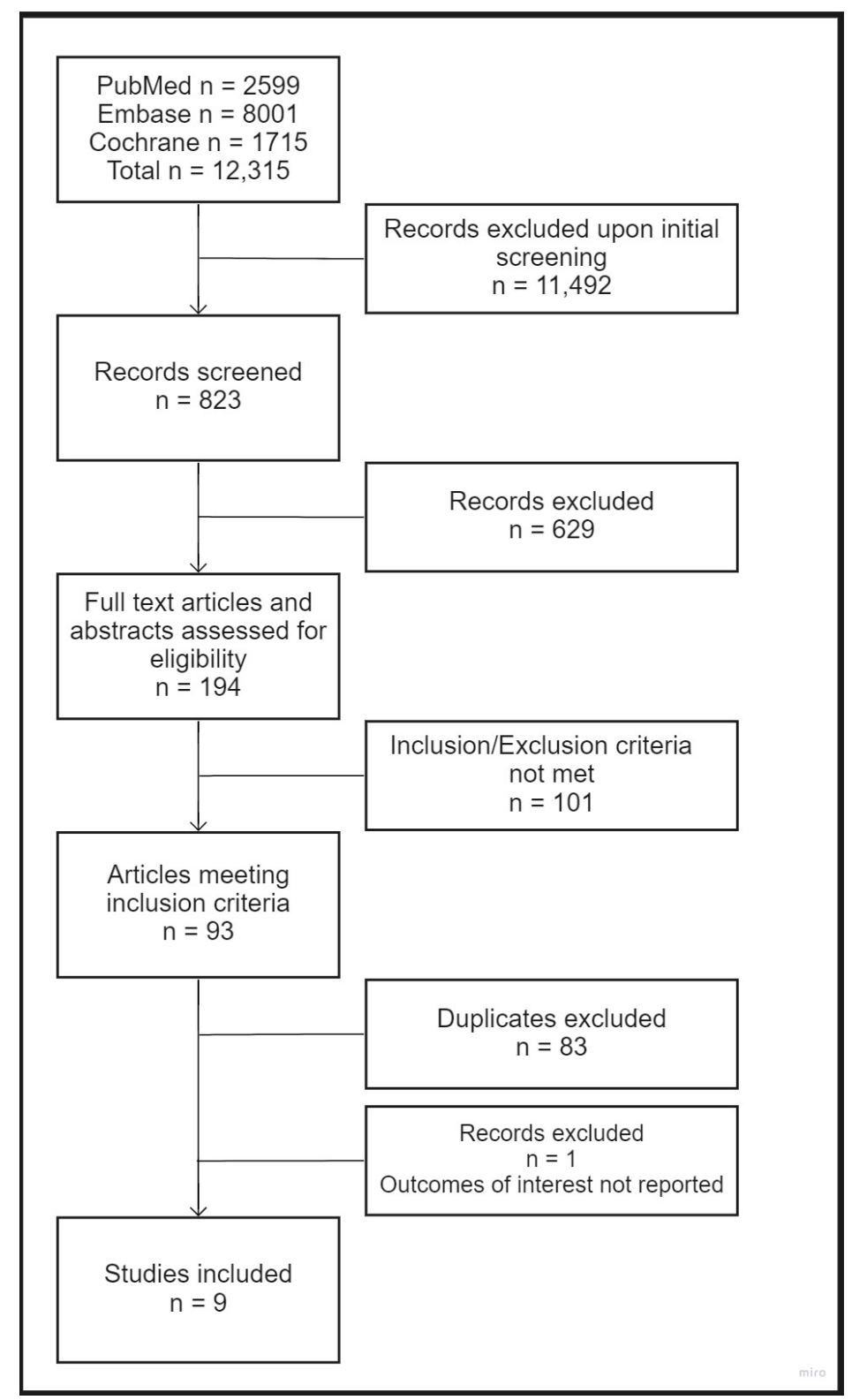

Figure 1. Flowchart of studies considered for inclusion.

Table 1. Design of phase III RCT in type 2 diabetes included in the NMA.

\begin{tabular}{|c|c|c|c|c|c|}
\hline $\begin{array}{c}\text { Study (Duration in } \\
\text { Weeks) }\end{array}$ & Design & Treatment Arms & Inclusion Criteria & $\begin{array}{l}\text { Primary } \\
\text { Endpoint }\end{array}$ & $\begin{array}{c}\text { Key } \\
\text { Secondary } \\
\text { Endpoints }\end{array}$ \\
\hline $\begin{array}{l}\text { LEAD-2 extension } \\
\text { (104) [32] }\end{array}$ & $\begin{array}{c}\text { Multinational, } \\
\text { randomized, parallel } \\
\text { assignment, } \\
\text { open-label, } \\
\text { active-comparator trial }\end{array}$ & $\begin{array}{l}\text { Lira } 0.6 \mathrm{mg} \text {, Lira } \\
1.2 \mathrm{mg} \text {, Lira } \\
1.8 \mathrm{mg} \text {, Glim } \\
4 \mathrm{mg} \text {, PLA }\end{array}$ & $\begin{array}{c}18-80 \text { years, diagnosed } \\
\text { with T2DM, on OAD } \\
\text { for }>3 \text { months, HbA1c } \\
7-11 \%, \text { BMI } \leq 40\end{array}$ & $\begin{array}{c}\text { Change in } \\
\text { HbA1c }\end{array}$ & $\begin{array}{c}\text { Change in } \\
\text { weight }\end{array}$ \\
\hline $\begin{array}{l}\text { LEAD-3 } \\
(52)[33]\end{array}$ & $\begin{array}{c}\text { Multicenter, } \\
\text { randomized, parallel } \\
\text { assignment, } \\
\text { double-blind, } \\
\text { double-dummy, } \\
\text { active-control trial }\end{array}$ & $\begin{array}{c}\text { Lira } 1.2 \mathrm{mg} \text {, Lira } \\
1.8 \mathrm{mg} \text {, Glim } \\
8 \mathrm{mg}\end{array}$ & $\begin{array}{c}\text { 18-80 years, diagnosed } \\
\text { with T2DM, treated } \\
\text { with diet/exercise or } \\
\text { with not more than } \\
\text { half maximal dose of } \\
\text { OAD for }>2 \text { months, } \\
\text { HbA1c } 7-11 \% \text {, } \\
\text { BMI } \leq 45\end{array}$ & $\begin{array}{c}\text { Change in } \\
\text { HbA1c }\end{array}$ & $\begin{array}{c}\text { Change in } \\
\text { weight }\end{array}$ \\
\hline
\end{tabular}


Table 1. Cont.

\begin{tabular}{|c|c|c|c|c|c|}
\hline $\begin{array}{l}\text { Study (Duration in } \\
\text { Weeks) }\end{array}$ & Design & $\begin{array}{l}\text { Treatment } \\
\text { Arms }\end{array}$ & Inclusion Criteria & $\begin{array}{l}\text { Primary } \\
\text { Endpoint }\end{array}$ & $\begin{array}{c}\text { Key } \\
\text { Secondary } \\
\text { Endpoints }\end{array}$ \\
\hline SUSTAIN-2 (56) [34] & $\begin{array}{c}\text { Multinational, } \\
\text { randomized, parallel } \\
\text { assignment, } \\
\text { double-blind, } \\
\text { active-comparator trial }\end{array}$ & $\begin{array}{c}\text { Sema QW } \\
0.5 \mathrm{mg} \text {, Sema } \\
\text { QW } 1 \mathrm{mg} \text {, Sita } \\
100 \mathrm{mg}\end{array}$ & $\begin{array}{c}\geq 18 \text { years, diagnosed with } \\
\text { T2DM, stable treatment } \\
\text { OAD for > } 3 \text { months (OAD: } \\
\text { MET, PIO, ROSI, or } \\
\text { combination), HbA1c } \\
7-10.5 \%\end{array}$ & $\begin{array}{l}\text { Change in } \\
\text { HbA1c }\end{array}$ & $\begin{array}{l}\text { Change in } \\
\text { weight }\end{array}$ \\
\hline SUSTAIN-3 (56) [19] & $\begin{array}{c}\text { Multinational, } \\
\text { randomized, parallel } \\
\text { assignment, } \\
\text { open-label, } \\
\text { active-comparator trial }\end{array}$ & $\begin{array}{c}\text { Sema QW } \\
1 \mathrm{mg}, \text { Exe } 2 \mathrm{mg}\end{array}$ & $\begin{array}{c}\geq 18 \text { years, diagnosed with } \\
\text { T2DM, 1-2 OAD for } \\
>3 \text { months (OAD: MET, } \\
\text { TZD, or SU), HbA1c } \\
7-10.5 \%\end{array}$ & $\begin{array}{l}\text { Change in } \\
\text { HbA1c }\end{array}$ & $\begin{array}{l}\text { Change in } \\
\text { weight }\end{array}$ \\
\hline $\begin{array}{l}\text { SUSTAIN-6 } \\
(104) \text { [35] }\end{array}$ & $\begin{array}{c}\text { Multinational, } \\
\text { randomized, parallel } \\
\text { assignment, } \\
\text { double-blind, } \\
\text { placebo-controlled } \\
\text { trial }\end{array}$ & $\begin{array}{l}\text { Sema QW } \\
0.5 \mathrm{mg}, \text { Sema } \\
\text { QW } 1 \mathrm{mg}, \\
2 \text { volume } \\
\text { matched PLA }\end{array}$ & $\begin{array}{l}\geq 50 \text { years, diagnosed with } \\
\text { T2DM, antidiabetic drug } \\
\text { naïve or on } 1-2 \text { OAD or } \\
\text { insulin (NPH, long acting } \\
\text { analogue, or premixed } \\
\text { insulin), both types of } \\
\text { insulin alone or with } \\
\text { combination } 1-2 \text { OAD, } \\
\text { HbA1c } \geq 7 \%\end{array}$ & $\begin{array}{l}\text { First } \\
\text { occurrence of } \\
\text { MACE }^{*}\end{array}$ & $\begin{array}{l}\text { Change in } \\
\text { HbA1c, change } \\
\text { in weight }\end{array}$ \\
\hline SCALE (56) [36] & $\begin{array}{c}\text { Multinational, } \\
\text { randomized, parallel } \\
\text { assignment, } \\
\text { double-blind, } \\
\text { placebo-controlled } \\
\text { trial }\end{array}$ & $\begin{array}{l}\text { Lira } 3 \text { mg, Lira } \\
1.8 \mathrm{mg} \text {, PLA }\end{array}$ & $\begin{array}{c}\geq 18 \text { years, diagnosed with } \\
\text { T2DM, treated with } \\
\text { diet/exercise or OAD } \\
\text { (OAD: MET, TZD, or SU) } \\
\text { or combination, HbA1c } \\
7-10 \%, \text { BMI } \geq 27\end{array}$ & $\begin{array}{l}\text { Change in } \\
\text { weight }\end{array}$ & $\begin{array}{l}\text { change in } \\
\text { HbA1c }\end{array}$ \\
\hline PIONEER-4 (52) [37] & $\begin{array}{c}\text { Multinational, } \\
\text { randomized, parallel } \\
\text { assignment, } \\
\text { double-blind, } \\
\text { double-dummy, } \\
\text { active-controlled and } \\
\text { placebo-controlled } \\
\text { trial }\end{array}$ & $\begin{array}{l}\text { Sema OD } 14 \\
\text { mg, Lira } \\
1.8 \mathrm{mg}, \text { PLA }\end{array}$ & $\begin{array}{l}\geq 18 \text { years, diagnosed with } \\
\text { T2DM, treated with MET } \\
\text { alone or in combination } \\
\text { with SGLT-2 inhibitor } \\
>3 \text { months, HbA1c } 7-9.5 \%\end{array}$ & $\begin{array}{l}\text { Change in } \\
\text { HbA1c }\end{array}$ & $\begin{array}{l}\text { Change in } \\
\text { weight }\end{array}$ \\
\hline $\begin{array}{l}\text { Pratley et al.; } \\
\quad \text { (52) [38] }\end{array}$ & $\begin{array}{c}\text { Multinational, } \\
\text { randomized, parallel } \\
\text { group, open-label, } \\
\text { active-comparator trial }\end{array}$ & $\begin{array}{l}\text { Lira } 1.2 \mathrm{mg}, \\
\text { Lira } 1.8 \mathrm{mg}, \\
\text { Sita } 100 \mathrm{mg}\end{array}$ & $\begin{array}{c}\geq 18 \text { years, diagnosed with } \\
\text { T2DM, treated with MET } \\
\text { for }>3 \text { months, HbA1c } \\
\quad 7.5-10 \%\end{array}$ & $\begin{array}{l}\text { Change in } \\
\text { HbAlc }\end{array}$ & - \\
\hline Kaku et al.; (56) [39] & $\begin{array}{c}\text { Multicenter, } \\
\text { single-country } \\
\text { randomized, parallel } \\
\text { group, } \\
\text { open-label, } \\
\text { active-controlled trial }\end{array}$ & $\begin{array}{l}\text { Sema QW } \\
0.5 \mathrm{mg} \text {, Sema } \\
\text { QW } 1 \mathrm{mg}, \\
\text { OAD (one } \\
\text { additional } \\
\text { OAD + } \\
\text { Pre-trial } \\
\text { treatment) }\end{array}$ & $\begin{array}{l}\geq 20 \text { years, diagnosed with } \\
\text { T2DM, treated with } \\
\text { diet/exercise for }>1 \text { month } \\
\text { or OAD monotherapy (SU, } \\
\text { Glinide, a-GI, TZD) for } \\
>2 \text { months, HbA1c } \\
7-10.5 \%\end{array}$ & $\begin{array}{l}\text { Emergent } \\
\text { adverse } \\
\text { events }\end{array}$ & $\begin{array}{l}\text { Change in } \\
\text { HbA1c, change } \\
\text { in weight }\end{array}$ \\
\hline
\end{tabular}

Lira = Liraglutide, Sema QW = Semaglutide once weekly, Sema OD = Semaglutide once daily, Glim = Glimepiride, Sita = Sitagliptin, $\mathrm{Exe}=$ exenatide, $\mathrm{OAD}=$ Oral anti-diabetic drugs, $\mathrm{PLA}=$ Placebo, $\mathrm{T} 2 \mathrm{DM}=$ Type 2 diabetes mellitus, $\mathrm{HbA1C}=$ Glycosylated hemoglobin, MET $=$ Metformin, $\mathrm{PIO}=$ Pioglitazone, $\mathrm{ROSI}=$ Rosiglitazone, $\mathrm{TZD}=$ Thiazolidinedione, $\mathrm{SU}=$ Sulfonylurea, $\mathrm{NPH}=\mathrm{Neutral}$ protamine Hagedorn, SGLT-2 = Sodium-glucose transport protein 2, a-GI = Alpha- glucosidase inhibitor, Glinide = Meglitinides, MACE = Major adverse cardiovascular events, $\mathrm{N} / \mathrm{R}=$ Not reported. ${ }^{*}$ MACE defined as cardiovascular death, non-fatal myocardial infarction, or nonfatal stroke. 
Table 2. Baseline characteristics of included studies.

\begin{tabular}{|c|c|c|c|c|c|c|c|c|c|}
\hline $\begin{array}{l}\text { Study (Duration; } \\
\text { Weeks) }\end{array}$ & Year & Arms & $\begin{array}{l}\text { Sample } \\
\text { Size }\end{array}$ & $\begin{array}{l}\text { Age in } \\
\text { Years } \\
\text { Means } \\
\text { (SD) }\end{array}$ & $\underset{\%}{\text { Male }}$ & $\begin{array}{c}\text { DOD } \\
\text { Means } \\
\text { (SD) }\end{array}$ & $\begin{array}{c}\text { HbA1c } \\
\text { Means } \\
\text { (SD) }\end{array}$ & $\begin{array}{l}\text { Weight } \\
\text { Means } \\
\text { (SD) }\end{array}$ & $\begin{array}{c}\text { BMI } \\
\text { Means } \\
\text { (SD) }\end{array}$ \\
\hline \multirow{5}{*}{$\begin{array}{c}\text { LEAD-2 } \\
\text { extension (104) }\end{array}$} & \multirow{5}{*}{2013} & Lira $0.6 \mathrm{mg}$ & \multirow{5}{*}{880} & $56(10.5)$ & 62.4 & $7(5)$ & $8.4(0.9)$ & $88(17)$ & $\mathrm{N} / \mathrm{R}$ \\
\hline & & Lira $1.2 \mathrm{mg}$ & & $57.2(9.2)$ & 53.8 & $7(5)$ & $8.3(0.9)$ & 88 (19) & $\mathrm{N} / \mathrm{R}$ \\
\hline & & Lira $1.8 \mathrm{mg}$ & & $56.8(9.4)$ & 58.7 & $8(5)$ & $8.3(0.9)$ & $88(16)$ & $\mathrm{N} / \mathrm{R}$ \\
\hline & & Glim 4 mg & & $57.3(8.8)$ & 57.4 & $8(5)$ & $8.4(0.9)$ & $89(17)$ & $\mathrm{N} / \mathrm{R}$ \\
\hline & & PLA & & $56(9.4)$ & 60 & $8(6)$ & $8.4(1)$ & $91(17)$ & $\mathrm{N} / \mathrm{R}$ \\
\hline \multirow{3}{*}{ LEAD-3 (52) } & \multirow{3}{*}{2009} & Lira $1.2 \mathrm{mg}$ & \multirow{3}{*}{746} & $53.7(11)$ & 46.6 & $5.2(5.5)$ & $8.3(1)$ & $92.5(19.2)$ & $33.2(5.6)$ \\
\hline & & Lira $1.8 \mathrm{mg}$ & & $52(10.8)$ & 49 & $5.3(5.1)$ & $8.3(1.1)$ & $92.8(20.7)$ & $32.8(6.3)$ \\
\hline & & Glim $8 \mathrm{mg}$ & & $53.4(10.9)$ & 53.6 & $5.6(5.1)$ & $8.4(1.2)$ & $93.4(19.2)$ & $33.2(5.6)$ \\
\hline \multirow{3}{*}{ SUSTAIN-2 (56) } & \multirow{3}{*}{2017} & Sema QW $0.5 \mathrm{mg}$ & \multirow{3}{*}{1225} & $54.8(10.2)$ & 51 & $6.44(4.7)$ & $8(0.92)$ & $89.93(20.39)$ & $32.43(6.2)$ \\
\hline & & Sema QW 1 mg & & $56(9.4)$ & 50 & $6.7(5.6)$ & $8(0.93)$ & $89.21(20.74)$ & $32.5(6.6)$ \\
\hline & & Sita $100 \mathrm{mg}$ & & $54.6(10.4)$ & 51 & $6.6(5.9)$ & $8.17(0.92)$ & 89.29 (19.67) & $32.45(5.8)$ \\
\hline \multirow{2}{*}{ SUSTAIN-3 (56) } & \multirow{2}{*}{2018} & Sema QW $1 \mathrm{mg}$ & \multirow{2}{*}{809} & $56.4(10.3)$ & 54.2 & $9(6)$ & $8.36(0.95)$ & $96.21(22.5)$ & $33.97(7.2)$ \\
\hline & & Exe $2 \mathrm{mg}$ & & $56.7(11.1)$ & 56.3 & $9.4(6.7)$ & $8.33(0.96)$ & $95.37(20.46)$ & $33.57(6.2)$ \\
\hline \multirow{4}{*}{ SUSTAIN-6 (104) } & \multirow{4}{*}{2016} & Sema QW0.5 mg & \multirow{4}{*}{3297} & $64.6(7.3)$ & 59.9 & $14.3(8.2)$ & $8.7(1.39)$ & $91.8(20.25)$ & $32.7(6.29)$ \\
\hline & & Sema QW $1 \mathrm{mg}$ & & $64.7(7.1)$ & 63 & $14.1(8.2)$ & $8.7(1.51)$ & $92.9(20.05)$ & $32.9(6.18)$ \\
\hline & & PLA 1 & & $64.8(7.6)$ & 58.5 & $14(8.5)$ & $8.7(1.49)$ & $91.8(20.35)$ & $32.9(6.35)$ \\
\hline & & PLA 2 & & $64.4(7.5)$ & 61.5 & $13.2(7.4)$ & $8.7(1.45)$ & $91.9(20.75)$ & $32.7(5.97)$ \\
\hline \multirow{3}{*}{ SCALE (56) } & \multirow{3}{*}{2015} & Lira 3 mg & \multirow{3}{*}{846} & $55(10.8)$ & 52 & $7.54(5.65)$ & $7.9(0.8)$ & 105.7 (21.9) & $37.1(6.5)$ \\
\hline & & Lira $1.8 \mathrm{mg}$ & & $54.9(10.7)$ & 51.2 & $7.43(5.16)$ & $8(0.8)$ & $105.8(21)$ & $37(6.9)$ \\
\hline & & PLA & & $54.7(9.8)$ & 45.8 & $6.71(5.07)$ & $7.9(0.8)$ & $106.5(21.3)$ & $37.4(7.1)$ \\
\hline \multirow{3}{*}{ PIONEER-4 (52) } & \multirow{3}{*}{2019} & Sema OD 14 mg & \multirow{3}{*}{711} & $56(10)$ & 52 & $7.8(5.7)$ & $8(0.7)$ & $92.9(20.6)$ & $32.5(5.9)$ \\
\hline & & Lira $1.8 \mathrm{mg}$ & & $56(10)$ & 52 & $7.3(5.3)$ & $8(0.7)$ & $95.5(21.9)$ & $33.4(6.7)$ \\
\hline & & PLA & & $57(10)$ & 52 & $7.8(5.5)$ & $7.9(0.7)$ & $93.2(20)$ & $32.9(6.1)$ \\
\hline \multirow{3}{*}{ Pratley et al. (52) } & \multirow{3}{*}{2011} & Lira $1.2 \mathrm{mg}$ & \multirow{3}{*}{497} & $55.9(9.6)$ & 51.6 & $6(4.5)$ & $8.4(0.8)$ & $\mathrm{N} / \mathrm{R}$ & $32.6(5.2)$ \\
\hline & & Lira $1.8 \mathrm{mg}$ & & $55(9.1)$ & 52.5 & $6.4(5.4)$ & $8.4(0.7)$ & $\mathrm{N} / \mathrm{R}$ & $33.1(5.1)$ \\
\hline & & Sita $100 \mathrm{mg}$ & & $55(9)$ & 54.8 & $6.3(5.4)$ & $8.5(0.7)$ & $\mathrm{N} / \mathrm{R}$ & $32.6(5.4)$ \\
\hline \multirow{3}{*}{ Kaku et al. (56) } & \multirow{3}{*}{2018} & Sema QW $0.5 \mathrm{mg}$ & \multirow{3}{*}{601} & $58(10.6)$ & 69.5 & $8.1(6)$ & $8(0.9)$ & $71(15.4)$ & $26.2(4.8)$ \\
\hline & & Sema QW $1 \mathrm{mg}$ & & $58.7(10.2)$ & 72.2 & $9.4(6.5)$ & $8.1(1)$ & $71.7(15.9)$ & $26.4(4.7)$ \\
\hline & & OAD & & $59.2(10.1)$ & 74.2 & $9.3(7)$ & $8.1(0.9)$ & $72.2(14.9)$ & $26.7(4.6)$ \\
\hline
\end{tabular}

Lira = Liraglutide, Sema QW = Semaglutide once weekly, Sema OD = Semaglutide once daily, Glim = Glimepiride, Sita = Sitagliptin, Exe $=$ exenatide, $\mathrm{OAD}=$ Oral anti-diabetic drugs, $\mathrm{PLA}=$ Placebo, HbA1C $=$ Glycosylated hemoglobin, N $/ \mathrm{R}=\mathrm{Not}$ reported, DOD $=$ duration of diabetes, $\mathrm{BMI}=$ body mass index, $\mathrm{SD}=$ standard deviation.

\subsection{Network Meta-Analysis Results}

Two outcomes were analysed and presented in this NMA. A random-effect, metaregression analysis was conducted on $\mathrm{HbA} 1 \mathrm{c}$ and weight for a better model fit. Significant treatment differences were found as shown in the matrix, Tables 3 and 4 . The evidence network for Hba1c and weight analysis shown in Figures 2 and 3, respectively.

$\mathrm{HbA1c}$ was reported in all nine studies. The $\mathrm{HbA1c}$ reduction from baseline was presented in the SUCRA score for ranking the main medications versus comparators, Table 5. Semaglutide $1 \mathrm{mg}$ scored the highest in $\mathrm{HbA1c}$ reduction, 90.5\% in SUCRA score, Table 5. As shown in the matrix, Table 3, semaglutide $1 \mathrm{mg}$ QW found to be significantly superior to liraglutide $0.6 \mathrm{mg}, 1.2 \mathrm{mg}, 1.8 \mathrm{mg}$ with a treatment difference of $0.56 \%, 0.47 \%$ and $0.3 \%$, respectively. Semaglutide $0.5 \mathrm{mg} \mathrm{QW}$ was found to be significantly superior to liraglutide $0.6 \mathrm{mg}$ and $1.2 \mathrm{mg}$ with a treatment difference of $0.25 \%$ and $0.17 \%$, respectively. The results of the NMA are presented as treatment differences in Figure 4.

Eight out of nine studies reported weight reduction. The weight reduction from baseline was presented in the SUCRA score for ranking the main medications versus comparators, Table 5. Semaglutide $1 \mathrm{mg}$ scored the highest in weight reduction, $84.9 \%$ in SUCRA score, Table 5. As shown in the matrix, Table 4, semaglutide $0.5 \mathrm{mg}$ and $1 \mathrm{mg} \mathrm{QW}$ were significantly associated with greater weight reduction than liraglutide $0.6 \mathrm{mg}$ with a treatment difference of $2.42 \mathrm{~kg}$ and $3.06 \mathrm{~kg}$, respectively. However, no significant reduction 
was found in comparison to liraglutide $1.2 \mathrm{mg}$ and $1.8 \mathrm{mg}$. The results of the NMA are presented as treatment differences in Figure 5.

Table 3. NMA matrix for $\mathrm{HbA1c}$ change from baseline: treatment difference.

\begin{tabular}{|c|c|c|c|c|c|c|c|c|c|c|c|c|}
\hline & Exe $2 \mathrm{mg}$ & $\begin{array}{l}\text { Glim } \\
4 \mathrm{mg}\end{array}$ & $\begin{array}{l}\text { Glim } \\
8 \mathrm{mg}\end{array}$ & $\begin{array}{c}\text { Lira } \\
0.6 \mathrm{mg}\end{array}$ & $\begin{array}{l}\text { Lira } \\
1.2 \mathrm{mg}\end{array}$ & $\begin{array}{l}\text { Lira } \\
1.8 \mathrm{mg}\end{array}$ & $\begin{array}{c}\text { Lira } \\
3 \mathrm{mg}\end{array}$ & OAD & PLA & $\begin{array}{c}\text { Sema } \\
\text { QW } \\
0.5 \mathrm{mg}\end{array}$ & $\begin{array}{c}\text { Sema } \\
\text { QW } \\
1 \mathrm{mg}\end{array}$ & $\begin{array}{c}\text { Sema } \\
\text { OD } \\
14 \mathrm{mg}\end{array}$ \\
\hline \multicolumn{13}{|l|}{ Exe $2 \mathrm{mg}$} \\
\hline Glim 4 mg & $\begin{array}{l}-0.203 \\
(0.428, \\
0.023)\end{array}$ & & & & & & & & & & & \\
\hline Glim 8 mg & $\begin{array}{l}0.250 \\
(0.015, \\
0.484)\end{array}$ & $\begin{array}{l}0.452 \\
(0.272, \\
0.632)\end{array}$ & & & & & & & & & & \\
\hline Lira $0.6 \mathrm{mg}$ & $\begin{array}{c}-0.063 \\
(-0.288 \\
0.163)\end{array}$ & $\begin{array}{c}0.140 \\
(-0.009 \\
0.289)\end{array}$ & $\begin{array}{l}-0.312 \\
(-0.492 \\
-0.132)\end{array}$ & & & & & & & & & \\
\hline Lira $1.2 \mathrm{mg}$ & $\begin{array}{c}-0.146 \\
(-0.348 \\
0.057)\end{array}$ & $\begin{array}{c}0.057 \\
(-0.077 \\
0.191)\end{array}$ & $\begin{array}{l}-0.395 \\
(-0.531 \\
-0.260)\end{array}$ & $\begin{array}{c}-0.083 \\
(-0.217 \\
0.051)\end{array}$ & & & & & & & & \\
\hline Lira $1.8 \mathrm{mg}$ & $\begin{array}{l}-0.315 \\
(-0.512 \\
-0.117)\end{array}$ & $\begin{array}{c}-0.112 \\
(-0.242, \\
0.018)\end{array}$ & $\begin{array}{l}-0.565 \\
(-0.700 \\
-0.429)\end{array}$ & $\begin{array}{l}-0.252 \\
(-0.382 \\
-0.122)\end{array}$ & $\begin{array}{c}-0.169 \\
(-0.253 \\
-0.086)\end{array}$ & & & & & & & \\
\hline Lira 3 mg & $\begin{array}{l}-0.528 \\
(-0.788 \\
-0.269)\end{array}$ & $\begin{array}{c}-0.326 \\
(-0.544, \\
-0.107)\end{array}$ & $\begin{array}{l}-0.778 \\
(-1.005 \\
-0.551)\end{array}$ & $\begin{array}{c}-0.466 \\
(-0.684, \\
-0.247)\end{array}$ & $\begin{array}{l}-0.383 \\
(-0.581, \\
-0.185)\end{array}$ & $\begin{array}{l}-0.214 \\
(-0.398 \\
-0.029)\end{array}$ & & & & & & \\
\hline OAD & $\begin{array}{c}0.682 \\
(0.480 \\
0.883)\end{array}$ & $\begin{array}{c}0.884 \\
(0.676 \\
1.093)\end{array}$ & $\begin{array}{l}0.432 \\
(0.213 \\
0.650)\end{array}$ & $\begin{array}{c}0.744 \\
(0.536 \\
0.953)\end{array}$ & $\begin{array}{c}0.827 \\
(0.643 \\
1.011)\end{array}$ & $\begin{array}{c}0.997 \\
(0.818 \\
1.175)\end{array}$ & $\begin{array}{l}1.210 \\
(0.965, \\
1.456)\end{array}$ & & & & & \\
\hline PLA & $\begin{array}{l}0.460 \\
(0.270, \\
0.649)\end{array}$ & $\begin{array}{l}0.662 \\
(0.527 \\
0.797)\end{array}$ & $\begin{array}{l}0.210 \\
(0.055, \\
0.365)\end{array}$ & $\begin{array}{l}0.522 \\
(0.387 \\
0.657)\end{array}$ & $\begin{array}{l}0.605 \\
(0.502 \\
0.709)\end{array}$ & $\begin{array}{c}0.775 \\
(0.687, \\
0.862)\end{array}$ & $\begin{array}{l}0.988 \\
(0.804, \\
1.172)\end{array}$ & $\begin{array}{l}-0.222 \\
(-0.391 \\
-0.053)\end{array}$ & & & & \\
\hline Sema $0.5 \mathrm{mg}$ & $\begin{array}{l}-0.317 \\
(-0.488 \\
-0.145)\end{array}$ & $\begin{array}{c}-0.114 \\
(-0.283, \\
0.055)\end{array}$ & $\begin{array}{l}-0.566 \\
(-0.748 \\
-0.385)\end{array}$ & $\begin{array}{l}-0.254 \\
(-0.423, \\
-0.085)\end{array}$ & $\begin{array}{l}-0.171 \\
(-0.309 \\
-0.033)\end{array}$ & $\begin{array}{c}-0.002 \\
(-0.132, \\
0.128)\end{array}$ & $\begin{array}{c}0.212 \\
(-0.001, \\
0.425)\end{array}$ & $\begin{array}{l}-0.998 \\
(-1.135 \\
-0.862)\end{array}$ & $\begin{array}{l}-0.776 \\
(-0.893 \\
-0.659)\end{array}$ & & & \\
\hline Sema $1 \mathrm{mg}$ & $\begin{array}{c}-0.620 \\
(-0.769 \\
-0.471)\end{array}$ & $\begin{array}{c}-0.417 \\
(-0.587, \\
-0.248)\end{array}$ & $\begin{array}{l}-0.870 \\
(-1.051, \\
-0.688)\end{array}$ & $\begin{array}{c}-0.557 \\
(-0.727, \\
-0.388)\end{array}$ & $\begin{array}{c}-0.474 \\
(-0.612, \\
-0.337)\end{array}$ & $\begin{array}{l}-0.305 \\
(-0.435 \\
-0.175)\end{array}$ & $\begin{array}{c}-0.092 \\
(-0.304, \\
0.121)\end{array}$ & $\begin{array}{l}-1.302 \\
(-1.438, \\
-1.165)\end{array}$ & $\begin{array}{l}-1.080 \\
(-1.197 \\
-0.963)\end{array}$ & $\begin{array}{c}-0.303 \\
(-0.389 \\
-0.218)\end{array}$ & & \\
\hline Sema 14 mg & $\begin{array}{l}-0.578 \\
(-0.806 \\
-0.349)\end{array}$ & $\begin{array}{l}-0.375 \\
(-0.555, \\
-0.195)\end{array}$ & $\begin{array}{l}-0.828 \\
(-1.017 \\
-0.638)\end{array}$ & $\begin{array}{l}-0.515 \\
(-0.695 \\
-0.335)\end{array}$ & $\begin{array}{l}-0.432 \\
(-0.586, \\
-0.279)\end{array}$ & $\begin{array}{l}-0.263 \\
(-0.399 \\
-0.127)\end{array}$ & $\begin{array}{c}-0.049 \\
(-0.270 \\
0.171)\end{array}$ & $\begin{array}{l}-1.259 \\
(-1.471 \\
-1.048)\end{array}$ & $\begin{array}{l}-1.037 \\
(-1.174 \\
-0.901)\end{array}$ & $\begin{array}{l}-0.261 \\
(-0.434, \\
-0.088)\end{array}$ & $\begin{array}{c}0.042 \\
(-0.131 \\
0.216)\end{array}$ & \\
\hline Sita $100 \mathrm{mg}$ & $\begin{array}{c}-0.369 \\
(-0.764 \\
0.033)\end{array}$ & $\begin{array}{c}0.571 \\
(0.901 \\
0.242)\end{array}$ & $\begin{array}{c}0.119 \\
(-0.050 \\
0.288)\end{array}$ & $\begin{array}{c}-0.431 \\
(-0.780 \\
0.090)\end{array}$ & $\begin{array}{l}-0.516 \\
(-0.771, \\
-0.263)\end{array}$ & $\begin{array}{c}-0.684 \\
(-0.931, \\
0.448)\end{array}$ & $\begin{array}{l}-0.894 \\
(-1.270 \\
-0.517)\end{array}$ & $\begin{array}{c}0.313 \\
(-0.042, \\
0.684)\end{array}$ & $\begin{array}{c}0.091 \\
(-0.151, \\
0.349)\end{array}$ & $\begin{array}{c}-0.684 \\
(-0.8 \\
-0.57)\end{array}$ & $\begin{array}{l}0.989 \\
(0.871 \\
1.106)\end{array}$ & $\begin{array}{c}-0.946 \\
(-0.777 \\
1.115)\end{array}$ \\
\hline
\end{tabular}

Lira = Liraglutide, Sema QW = Semaglutide once weekly, Sema OD = Semaglutide once daily, Glim = Glimepiride, Sita = Sitagliptin,

Exe $=$ exenatide, $\mathrm{OAD}=$ Oral anti-diabetic drugs, $\mathrm{PLA}=$ Placebo, $\mathrm{HbA1C}=$ Glycosylated hemoglobin, Not significant if crosses zero.

Table 4. NMA matrix for weight change from baseline: treatment difference.

\begin{tabular}{|c|c|c|c|c|c|c|c|c|c|c|c|}
\hline & Exe $2 \mathrm{mg}$ & $\begin{array}{l}\text { Glim } \\
4 \mathrm{mg}\end{array}$ & $\begin{array}{l}\text { Glim } \\
8 \mathrm{mg}\end{array}$ & $\begin{array}{c}\text { Lira } \\
0.6 \mathrm{mg} \\
\end{array}$ & $\begin{array}{c}\text { Lira } \\
1.2 \mathrm{mg}\end{array}$ & $\begin{array}{c}\text { Lira } \\
1.8 \mathrm{mg}\end{array}$ & Lira $3 \mathrm{mg}$ & OAD & PLA & $\begin{array}{c}\text { Sema QW } \\
0.5 \mathrm{mg}\end{array}$ & $\begin{array}{c}\text { Sema QW } \\
1 \mathrm{mg}\end{array}$ \\
\hline Glim 4 mg & $\begin{array}{c}0.90 \\
(-6.80 \\
8.72)\end{array}$ & & & & & & & & & & \\
\hline Glim 8 mg & $\begin{array}{c}-1.85 \\
(-10.17 \\
6.37)\end{array}$ & $\begin{array}{c}-0.86 \\
(-7.26 \\
5.32)\end{array}$ & & & & & & & & & \\
\hline Lira $0.6 \mathrm{mg}$ & $\begin{array}{c}-1.74 \\
(-4.64 \\
3.98)\end{array}$ & $\begin{array}{c}0.81 \\
(-2.35 \\
0.93)\end{array}$ & $\begin{array}{c}0.86 \\
(-0.37 \\
1.14)\end{array}$ & & & & & & & & \\
\hline Lira $1.2 \mathrm{mg}$ & $\begin{array}{c}-1.02 \\
(-3.99 \\
1.95)\end{array}$ & $\begin{array}{c}-1.08 \\
(-2.77 \\
0.56)\end{array}$ & $\begin{array}{c}-0.80 \\
(-1.74 \\
0.51)\end{array}$ & $\begin{array}{c}-1.31 \\
(-5.85 \\
3.36)\end{array}$ & & & & & & & \\
\hline Lira $1.8 \mathrm{mg}$ & $\begin{array}{c}-1.36 \\
(-2.35 \\
1.47)\end{array}$ & $\begin{array}{c}-2.45 \\
(-4.03 \\
0.13)\end{array}$ & $\begin{array}{c}-1.54 \\
(-1.93 \\
1.11)\end{array}$ & $\begin{array}{c}-1.63 \\
(-6.23 \\
2.85)\end{array}$ & $\begin{array}{c}-0.34 \\
(-3.32 \\
2.58)\end{array}$ & & & & & & \\
\hline Lira $3 \mathrm{mg}$ & $\begin{array}{c}-1.66 \\
(-14.68 \\
1.65)\end{array}$ & $\begin{array}{c}-2.66 \\
(-4.89 \\
0.78)\end{array}$ & $\begin{array}{c}-2.73 \\
(-4.55 \\
1.90)\end{array}$ & $\begin{array}{c}-2.85 \\
(-6.26 \\
3.37)\end{array}$ & $\begin{array}{c}-1.56 \\
(-7.13 \\
3.78)\end{array}$ & $\begin{array}{c}-1.22 \\
(-6.08 \\
3.57)\end{array}$ & & & & & \\
\hline OAD & $\begin{array}{c}0.89 \\
(-1.04 \\
4.20)\end{array}$ & $\begin{array}{c}1.93 \\
(-5.20 \\
9.16)\end{array}$ & $\begin{array}{c}1.09 \\
(-6.44, \\
8.48)\end{array}$ & $\begin{array}{c}-0.83 \\
(-8.14 \\
6.41)\end{array}$ & $\begin{array}{c}-2.09 \\
(-8.55 \\
4.28)\end{array}$ & $\begin{array}{c}2.45 \\
(-3.76 \\
8.67)\end{array}$ & $\begin{array}{l}-3.65 \\
(-3.56 \\
11.11)\end{array}$ & & & & \\
\hline
\end{tabular}


Table 4. Cont.

\begin{tabular}{|c|c|c|c|c|c|c|c|c|c|c|c|}
\hline & Exe $2 \mathrm{mg}$ & $\begin{array}{l}\text { Glim } \\
4 \mathrm{mg}\end{array}$ & $\begin{array}{l}\text { Glim } \\
8 \mathrm{mg}\end{array}$ & $\begin{array}{c}\text { Lira } \\
0.6 \mathrm{mg}\end{array}$ & $\begin{array}{l}\text { Lira } \\
1.2 \mathrm{mg}\end{array}$ & $\begin{array}{c}\text { Lira } \\
1.8 \mathrm{mg}\end{array}$ & Lira $3 \mathrm{mg}$ & OAD & PLA & $\begin{array}{l}\text { Sema QW } \\
0.5 \mathrm{mg}\end{array}$ & $\begin{array}{l}\text { Sema QW } \\
1 \mathrm{mg}\end{array}$ \\
\hline PLA & $\begin{array}{c}0.31 \\
(-4.30 \\
4.93)\end{array}$ & $\begin{array}{c}1.32 \\
(-3.32 \\
6.06)\end{array}$ & $\begin{array}{c}0.48 \\
(-5.06 \\
5.95)\end{array}$ & $\begin{array}{c}1.45 \\
(-3.20 \\
6.08)\end{array}$ & $\begin{array}{c}2.72 \\
(-1.09 \\
6.43)\end{array}$ & $\begin{array}{c}3.09 \\
(-0.20 \\
6.32)\end{array}$ & $\begin{array}{c}4.30 \\
(-0.57 \\
9.20)\end{array}$ & $\begin{array}{c}0.64 \\
(-6.42 \\
5.30)\end{array}$ & & & \\
\hline Sema $0.5 \mathrm{mg}$ & $\begin{array}{l}-2.13 \\
(-4.15 \\
-1.89)\end{array}$ & $\begin{array}{l}-4.19 \\
(-8.16 \\
-2.62)\end{array}$ & $\begin{array}{c}1.97 \\
(-4.34 \\
8.22)\end{array}$ & $\begin{array}{l}-2.42 \\
(-6.22 \\
-1.44)\end{array}$ & $\begin{array}{c}-0.89 \\
(-3.60 \\
3.97)\end{array}$ & $\begin{array}{c}-0.27 \\
(-4.59 \\
4.29)\end{array}$ & $\begin{array}{c}-0.48 \\
(-3.51 \\
3.73)\end{array}$ & $\begin{array}{l}-4.25 \\
(-3.53 \\
-6.13)\end{array}$ & $\begin{array}{l}-3.84 \\
(-2.09 \\
-5.94)\end{array}$ & & \\
\hline Sema $1 \mathrm{mg}$ & $\begin{array}{l}-3.80 \\
(-4.60 \\
-2.96)\end{array}$ & $\begin{array}{l}-4.03 \\
(-4.97 \\
-3.07)\end{array}$ & $\begin{array}{l}-2.68 \\
(-3.58 \\
-1.77)\end{array}$ & $\begin{array}{l}-3.06 \\
(-6.02, \\
-0.82,)\end{array}$ & $\begin{array}{c}-1.23 \\
(-4.07 \\
3.68)\end{array}$ & $\begin{array}{c}-1.58 \\
(-4.16 \\
2.94)\end{array}$ & $\begin{array}{c}-0.82 \\
(-3.36 \\
3.00)\end{array}$ & $\begin{array}{l}-4.85 \\
(-5.62, \\
-3.88,)\end{array}$ & $\begin{array}{l}-4.04 \\
(-5.61 \\
-2.47)\end{array}$ & $\begin{array}{c}-0.35 \\
(-3.59,3.29)\end{array}$ & \\
\hline Sita 100 mg & $\begin{array}{c}1.06 \\
(-1.58 \\
1.50)\end{array}$ & $\begin{array}{c}1.09 \\
(-1.47 \\
1.85)\end{array}$ & $\begin{array}{c}0.19 \\
(-2.55 \\
2.16)\end{array}$ & $\begin{array}{c}1.32 \\
(-4.24 \\
7.01)\end{array}$ & $\begin{array}{c}0.01 \\
(-4.13 \\
4.33)\end{array}$ & $\begin{array}{c}0.31 \\
(-3.61, \\
4.35)\end{array}$ & $\begin{array}{c}1.51 \\
(-4.39 \\
7.61)\end{array}$ & $\begin{array}{l}-2.16 \\
(-7.98 \\
3.86)\end{array}$ & $\begin{array}{c}-2.77 \\
(-6.98 \\
1.60)\end{array}$ & $\begin{array}{c}0.25(-2.38 \\
2.85)\end{array}$ & $\begin{array}{c}0.90(-3.19 \\
5.01)\end{array}$ \\
\hline
\end{tabular}

Lira = Liraglutide, Sema QW = Semaglutide once weekly, Sema OD = Semaglutide once daily, Glim = Glimepiride, Sita = Sitagliptin Exe $=$ exenatide, $\mathrm{OAD}=$ Oral anti-diabetic drugs, PLA = Placebo, Not significant if crosses zero.

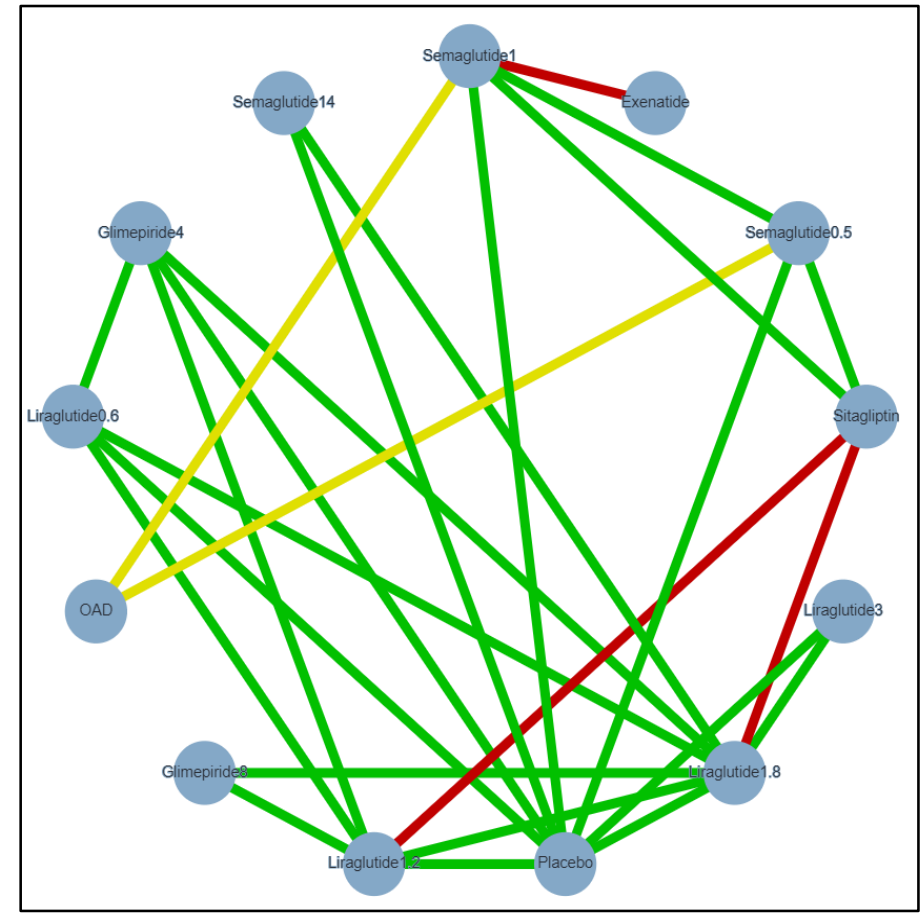

Figure 2. Evidence network of included studies for $\mathrm{HbA} 1 \mathrm{c}$ analysis. Semaglutide $1=$ Semaglutide $1 \mathrm{mg}$ once-weekly (QW), Semaglutide 0.5 = Semaglutide $0.5 \mathrm{mg} \mathrm{QW}$, Semaglutide $14=$ oral semaglutide $14 \mathrm{mg}$, Glimpiride 4 = Glimepiride $4 \mathrm{mg}$, Glimpiride 8 = Glimepiride $8 \mathrm{mg}$, Liraglutide 0.6 = Liraglutide $0.6 \mathrm{mg}$ once-daily (OD), Liraglutide $1.2=$ Liraglutide $1.2 \mathrm{mg}$ OD, Liraglutide 1.8 = Liraglutide $1.8 \mathrm{mg}$ OD, Liraglutide $3=$ Liraglutide $3 \mathrm{mg}$ OD, OAD = Oral antidiabetic drugs, Exenatide = Exenatide $2 \mathrm{mg}$, Sitagliptin = Sitagliptin $100 \mathrm{mg}, \mathrm{HbA} 1 \mathrm{c}=$ glycated hemoglobin, Green: Low risk of bias, Yellow: Unclear risk of bias, Red: High risk of bias. 


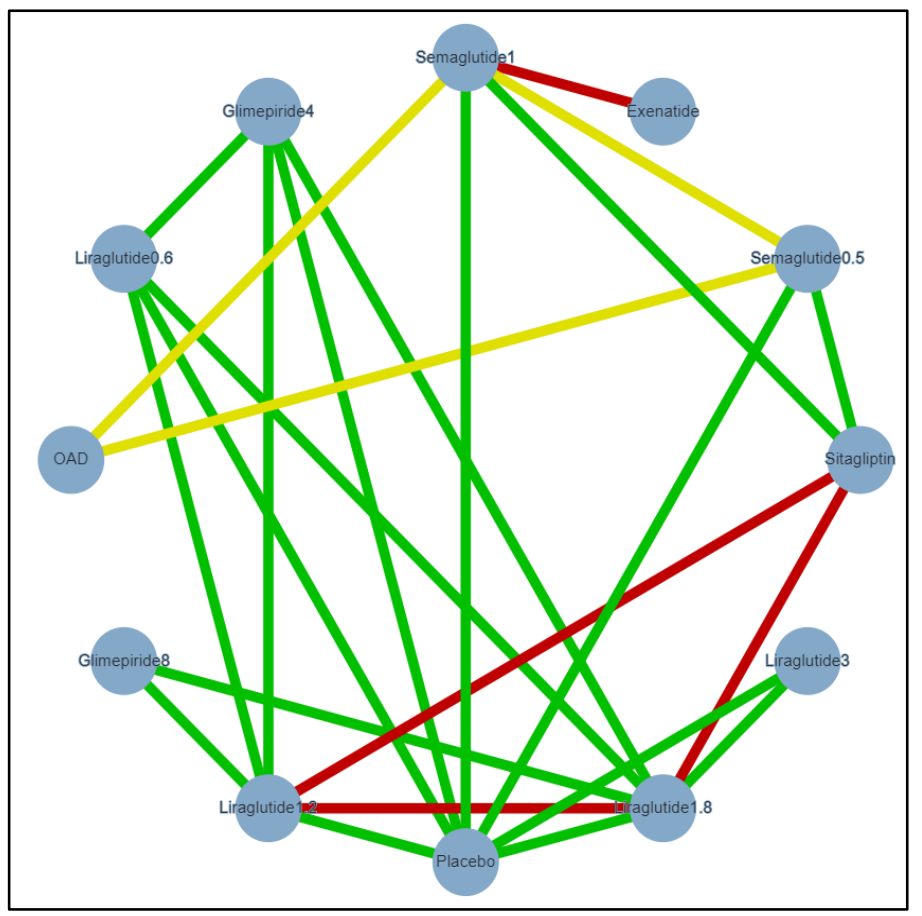

Figure 3. Evidence network of included studies for weight analysis. Semaglutide $1=$ Semaglutide $1 \mathrm{mg}$ once-weekly (QW), Semaglutide $0.5=$ Semaglutide $0.5 \mathrm{mg}$ QW, Glimpiride 4 = Glimepiride $4 \mathrm{mg}$, Glimpiride 8 = Glimepiride $8 \mathrm{mg}$, Liraglutide 0.6 = Liraglutide $0.6 \mathrm{mg}$ once-daily (OD), Liraglutide 1.2 = Liraglutide $1.2 \mathrm{mg}$ OD, Liraglutide 1.8 = Liraglutide $1.8 \mathrm{mg}$ OD, Liraglutide 3 = Liraglutide $3 \mathrm{mg}$ OD, $\mathrm{OAD}=$ Oral anti-diabetic drugs, Exenatide = Exenatide $2 \mathrm{mg}$, Sitagliptin = Sitagliptin $100 \mathrm{mg}$, Green: Low risk of bias, Yellow: Unclear risk of bias, Red: High risk of bias.

Table 5. Surface under the cumulative ranking curve (SUCRA) values for each intervention.

\begin{tabular}{ccc}
\hline Treatment & HbA1c & Weight \\
\hline Semaglutide 1 mg QW & 0.9055 & 0.8486 \\
Semaglutide 0.5 mg QW & 0.7423 & 0.7308 \\
Semaglutide 14 mg OD & 0.7377 & 0.7193 \\
Liraglutide 1.8 mg OD & 0.7431 & 0.7165 \\
Liraglutide 1.2 mg OD & 0.7029 & 0.5340 \\
Liraglutide 3 mg OD & 0.5137 & 0.4344 \\
Sitagliptin 100 mg & 0.5039 & 0.4122 \\
Liraglutide 0.6 mg OD & 0.4514 & 0.4019 \\
Glimepiride 4 mg & 0.4083 & 0.3426 \\
Glimepiride 8 mg & 0.3237 & 0.3161 \\
OAD & 0.2198 & 0.2085 \\
Exenatide 2 mg & 0.2157 & 0.0975 \\
\hline
\end{tabular}

$\mathrm{QW}=$ once weekly, $\mathrm{OD}=$ once daily, $\mathrm{OAD}=$ Oral anti-diabetic drugs, HbA1C = Glycosylated hemoglobin. Green indicate highest score, blue is the second highest, and grey is not included. 


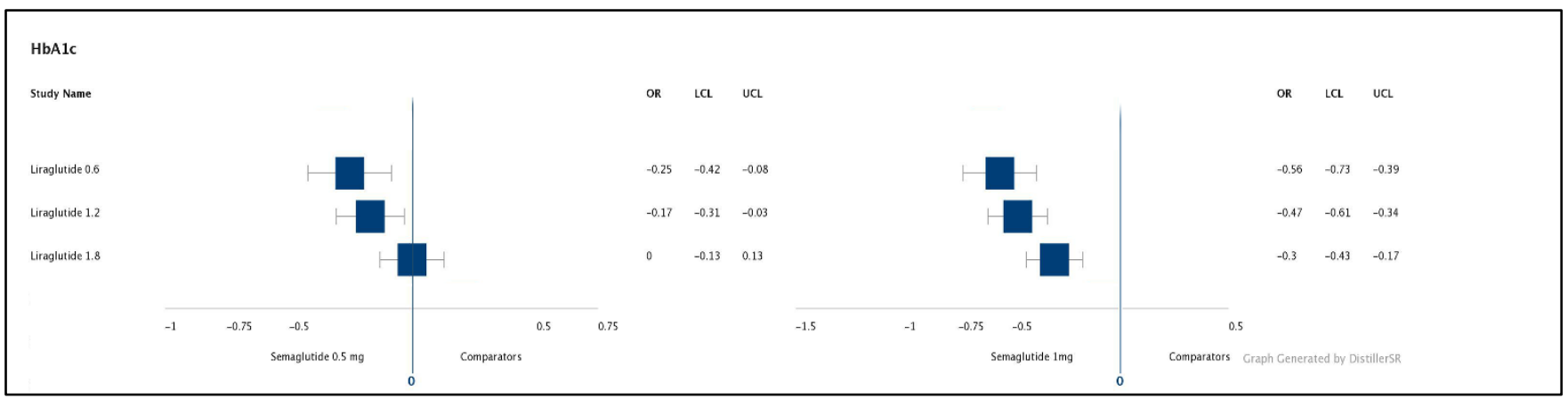

Figure 4. Forest plots of the NMA results: HbA1c outcomes for the primary comparators. The NMA results are presented as forest plots for a change from baseline in $\mathrm{HbA1c}$. OR = treatment difference, LCL and UCL = lower and upper credible intervals $(95 \% \mathrm{Crl})$ for mean treatment effect. Treatment differences are considered significant when the $95 \% \mathrm{CrI}$ excludes 0. $\mathrm{HbA} 1 \mathrm{c}=$ glycated hemoglobin, NMA = network meta-analysis.

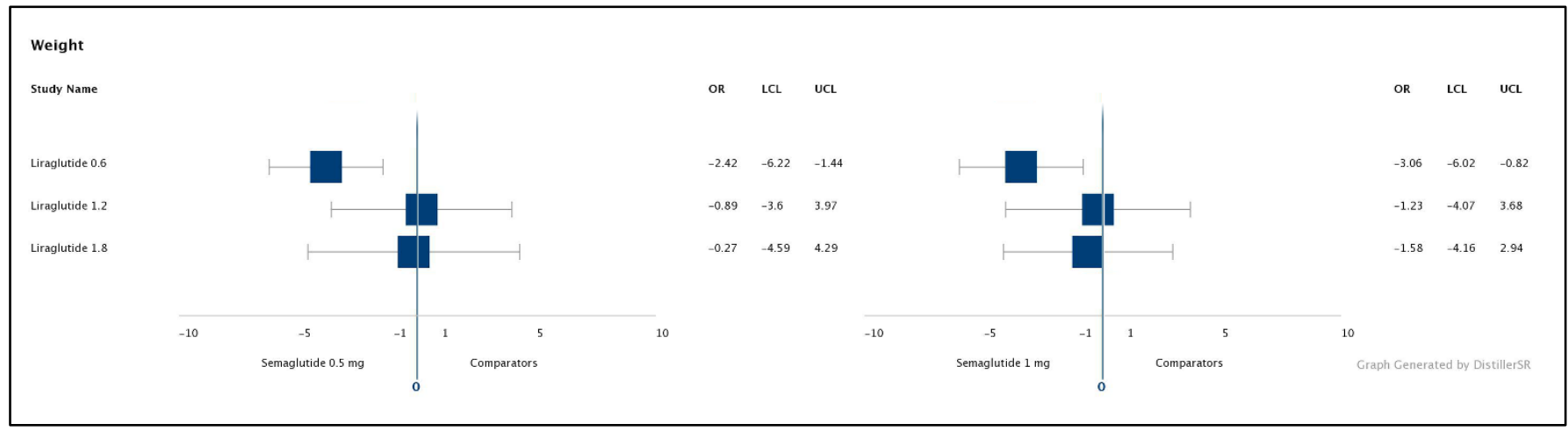

Figure 5. Forest plots of the NMA results: weight outcomes for primary comparators. The NMA results are presented as forest plots for a change from baseline in weight. OR = treatment difference, LCL and UCL = lower and upper credible intervals $(95 \% \mathrm{Crl})$ for mean treatment effect. Treatment differences are considered significant when the $95 \% \mathrm{CrI}$ excludes 0 . NMA = network meta-analysis.

\section{Discussion}

This network meta-analysis aimed to prove the long-term effects of semaglutide QW versus liraglutide $\mathrm{OD}$ on $\mathrm{HbA} 1 \mathrm{c}$ and weight change in patients with T2DM. To our knowledge, no other NMA explored the long-term efficacy of semaglutide versus liraglutide. However, several reviews reported the early effects of semaglutide and liraglutide on $\mathrm{HbA1c}$ and weight change.

Dose-dependent effect was seen in all the doses of interest, that is, semaglutide $(0.5 \mathrm{mg}$, $1 \mathrm{mg}$ ) and liraglutide $(1.2 \mathrm{mg}, 1.8 \mathrm{mg})$. While, liraglutide $3 \mathrm{mg}$ was included in a single RCT as part of this NMA, this might have led to the wide credible intervals and no significance.

In an NMA published in 2019 by Mishriky et al., semaglutide QW with other GLP-1 RA and dipeptidyl peptidase-4 inhibitors (DPP-4i) was compared in five trials with a duration of $\geq 12$ weeks, where they found that semaglutide $1 \mathrm{mg}$ was significantly superior in reducing $\mathrm{HbA} 1 \mathrm{c}$, with a change of $-0.38 \%$ and $-1.14 \%$, respectively [40]. Additionally, a meta-analyses (MA) by Shi et al. focused on semaglutide RCTs found that semaglutide was more effective in glycemic control in comparison to exenatide and dulaglutide, with a significant difference of $-0.47 \%$ [41]. However, they reported high heterogeneity $\left(\mathrm{I}^{2}=92 \%\right)$ with regard to the study duration and dosage used [41]. Another MA published by Li et al. also compared semaglutide to placebo and other active comparators including exenatide and dulaglutide, where semaglutide showed further reduction of $\mathrm{HbA} 1 \mathrm{c}$ with a change of $0.85 \%$ [42]. However, as disclosed in the Limitations section, the analysis may be restricted due to the significant heterogeneity $\left(\mathrm{I}^{2}=94 \%\right)$, as the analysis included trials ranging from 
12 weeks up to 104 weeks [42]. Furthermore, both MA used only direct comparison in their model. Thus, liraglutide was not included in the aforementioned MAs [41,42]. Our analysis suggested superiority on the long-term effects of semaglutide $1 \mathrm{mg}$ QW over liraglutide OD, based on studies with a duration of $\geq 52$ weeks, as shown by the SUCRA score in Table 5.

In an NMA published by Witkowski et al., semaglutide $1 \mathrm{mg}$ QW was the most effective compared to other GLP-1 RA in reducing weight in $24 \pm 4$-week trials [21]. Again, our analysis showed constant superiority of semaglutide $1 \mathrm{mg}$ QW over liraglutide OD even with longer-term use, as shown in the SUCRA score in Table 5. In a phase II trial by Nauck et al., comparing a 12-week weight change of semaglutide versus liraglutide, they reported statistically significant weight reduction with semaglutide versus liraglutide [43]. Both doses of once-weekly semaglutide $0.8 \mathrm{mg}$ and $1.6 \mathrm{mg}$ were greater in weight reduction compared to liraglutide $1.8 \mathrm{mg}$ OD. However, doses of semaglutide used in the trial were experimental non-FDA approved. Additionally, the dose escalation protocol varied between the arms of semaglutide. The reported superiority of semaglutide $0.8 \mathrm{mg}$ QW compared to liraglutide $1.8 \mathrm{mg}$ was found to be consistent with the semaglutide $1 \mathrm{mg}$ QW and semaglutide $0.5 \mathrm{mg}$ QW generated in the SUCRA score of this NMA, Table 5. In the recently published SUSTAIN 10, a phase $3 \mathrm{~b}$ trial, liraglutide $1.2 \mathrm{mg}$ was compared against subcutaneous semaglutide $1 \mathrm{mg}$ over a duration of 30 weeks. Semaglutide $1 \mathrm{mg}$ showed significant superiority over liraglutide $1.2 \mathrm{mg}$ with a treatment difference of $0.69 \%$ in $\mathrm{HbA} 1 \mathrm{c}$ reduction. Due to the short duration of the trial, the presumed long-term effect of semaglutide was not reached as mentioned in the limitation section of the study [44]. Nevertheless, treatment differences reported in SUSTAIN 10 was supportive of this NMA results. An NMA by Webb et al. included long-term RCTs reported significant superiority of injectable semaglutide over other GLP-1 RAs including liraglutide. However, this NMA included only Japanese population using the Japanese protocol for liraglutide, a maximum dose of liraglutide $0.9 \mathrm{mg}$, which is not the recommended therapeutic dose by the manufacturing pharmaceutical company, and the U.S. FDA [14]. Additionally, the number of analyzed trials was considerably low, only four, with only a single trial including the therapeutic dose of semaglutide [45].

The strengths of this NMA includes the quality of the analyzed RCTs. In addition, to ensure all relevant RCTs were included, a through systematic literature review was conducted. Furthermore, the robustness of the results and conclusions were demonstrated across several sensitivity and restricted analyses. Exploratory meta-regression analyses also validated the choice model used for the key analyses, change in $\mathrm{HbA} 1 \mathrm{c}$ and weight. Some common limitations were faced during this NMA, affecting the heterogeneity in the overall risk of bias. Analyses included different study designs, open-label, double-blind, and extension trials. In addition, there were variabilities in completion rates among the trials. Additionally, the low number of RCTs included in the NMA, a total of nine trials. Furthermore, some of the included RCTs were funded by the manufacturing pharmaceutical company. Moreover, this NMA included studies with multiple races and ethnicities while, no specific analysis was conducted regarding their distribution.

\section{Conclusions}

To date, no long-term phase III trials directly comparing semaglutide QW and liraglutide OD are available. Thus, the estimates driven from this NMA provide valuable evidence in the decision-making process for patients with T2DM. This NMA illustrated that semaglutide could improve the control of blood glucose and body weight. The capacity of long-term glycemic control and body weight reduction of injectable semaglutide appears to be more effective than other GLP-1 RAs, including liraglutide. However, considering the number of included studies and potential limitations, more large-scale, head-to-head, well-designed RCTs are needed to confirm these findings. 
Author Contributions: H.A.A., I.F.A., T.J.A., A.M.B.R. and A.M.T. helped with the design of the study protocol. Data extraction done by H.A.A. and I.F.A. independently. Statistical analysis and NMA model were done by W.A.-M. Manuscript draft was written by H.A.A. and I.F.A. Manuscript revised by A.M.T., T.J.A. and A.M.B.R. H.A.A. and I.F.A. equally contributed in producing the manuscript. All authors have read and agreed to the published version of the manuscript.

Funding: This research received no external funding.

Institutional Review Board Statement: Not applicable.

Informed Consent Statement: Not applicable.

Data Availability Statement: The datasets generated and/or analyzed during the current study are available from the corresponding author on reasonable request.

Acknowledgments: We thank Mostafa F. Abbas for his great support in protocol submission, ethical approval process and guidance.

Conflicts of Interest: The authors declare no conflict of interest.

Registration: This is a sub-study of the protocol registered in PROSPERO, number CRD42018091598.

\section{References}

1. Stumvoll, M.; Goldstein, B.J.; van Haeften, T.W. Type 2 diabetes: Principles of pathogenesis and therapy. Lancet 2005, 365, 1333-1346. [CrossRef]

2. Association, A.D. Standards of Medical Care in Diabetes-2016: Summary of Revisions. Diabetes Care 2016, 39 (Suppl. 1), S4-S5.

3. Zoungas, S.; Chalmers, J.; Ninomiya, T.; Li, Q.; Cooper, M.E.; Colagiuri, S.; Fulcher, G.; De Galan, B.E.; Harrap, S.; Hamet, P.; et al. Association of $\mathrm{HbA} 1 \mathrm{c}$ levels with vascular complications and death in patients with type 2 diabetes: Evidence of glycaemic thresholds. Diabetologia 2012, 55, 636-643. [CrossRef]

4. UK Prospective Diabetes Study (UKPDS) Group. Effect of intensive blood-glucose control with metformin on complications in overweight patients with type 2 diabetes (UKPDS 34). Lancet 1998, 352, 854-865. [CrossRef]

5. UK Prospective Diabetes Study (UKPDS) Group. UKPDS 28: A randomized trial of efficacy of early addition of metformin in sulfonylurea-treated type 2 diabetes. Diabetes Care 1998, 21, 87-92. [CrossRef]

6. Aroda, V.R. A review of GLP-1 receptor agonists: Evolution and advancement, through the lens of randomised controlled trials. Diabetes Obes. Metab. 2018, 20 (Suppl. 1), 22-33. [CrossRef] [PubMed]

7. Guja, C.; Miulescu, R.D. Semaglutide-the "new kid on the block" in the field of glucagonlike peptide-1 receptor agonists? Ann. Transl. Med. 2017, 5, 23. [CrossRef] [PubMed]

8. Chatterjee, S.; Davies, M.J.; Khunti, K. What have we learnt from "real world" data, observational studies and meta-analyses. Diabetes Obes. Metab. 2018, 20 (Suppl. 1), 47-58. [CrossRef] [PubMed]

9. Dhillon, S. Semaglutide: First Global Approval. Drugs 2018, 78, 275-284. [CrossRef]

10. Sharma, A.K. Albiglutide: Is a better hope against diabetes mellitus? Biomed. Pharmacother. 2018, 77, 120-128. [CrossRef]

11. Administration. 2016 Novel Drugs Summary. 2017. Available online: https://www.fda.gov/downloads/drugs/ developmentapprovalprocess/druginnovation/ucm536693.pdf (accessed on 1 January 2021).

12. Administration, Dulaglutide. 2017. Available online: https://www.accessdata.fda.gov/drugsatfda_docs/label/2017/125469s011 s013lbl.pdf\#page $=21$ (accessed on 1 January 2021).

13. Harris, B.K.; McCarty, D.J. Efficacy and tolerability of glucagon-like peptide-1 receptor agonists in patients with type 2 diabetes mellitus. Adv. Endocrinol. Metab. 2015, 6, 3-18. [CrossRef] [PubMed]

14. Fda.gov. Available online: https://www.accessdata.fda.gov/drugsatfda_docs/label/2020/022341s036lbl.pdf (accessed on 17 August 2021).

15. Trujillo, M.J.; Nuffer, W.; Ellis, S.L. GLP-1 receptor agonists: A review of head-to-head clinical studies. Adv. Endocrinol. Metab. 2015, 6, 19-28. [CrossRef]

16. Madsbad, S. Review of head-to-head comparisons of glucagon-like peptide-1 receptor agonists. Diabetes Obes. Metab. 2016, 18, 317-332. [CrossRef] [PubMed]

17. Sorli, C.; Harashima, S.I.; Tsoukas, G.M.; Unger, J.; Karsbøl, J.D.; Hansen, T.; Bain, S.C. Efficacy and safety of once-weekly semaglutide monotherapy versus placebo in patients with type 2 diabetes (SUSTAIN 1): A double-blind, randomised, placebocontrolled, parallel-group, multinational, multicentre phase 3a trial. Lancet Diabetes Endocrinol. 2017, 5, 251-260. [CrossRef]

18. Pratley, R.E.; Aroda, V.R.; Lingvay, I.; Lüdemann, J.; Andreassen, C.; Navarria, A.; Viljoen, A. Semaglutide versus dulaglutide once weekly in patients with type 2 diabetes (SUSTAIN 7): A randomised, open-label, phase $3 \mathrm{~b}$ trial. Lancet Diabetes Endocrinol. 2018, 6, 275-286. [CrossRef]

19. Ahmann, A.J.; Capehorn, M.; Charpentier, G.; Dotta, F.; Henkel, E.; Lingvay, I.; Holst, A.G.; Annett, M.P.; Aroda, V.R. Efficacy and Safety of Once-Weekly Semaglutide Versus Exenatide ER in Subjects With Type 2 Diabetes (SUSTAIN 3): A 56-Week, Open-Label, Randomized Clinical Trial. Diabetes Care 2018, 41, 258-266. [CrossRef] [PubMed] 
20. Courtney, H.; Nayar, R.; Rajeswaran, C.; Jandhyala, R. Long-term management of type 2 diabetes with glucagon-like peptide-1 receptor agonists. Diabetes Metab. Syndr. Obes. 2017, 10, 79-87. [CrossRef]

21. Witkowski, M.; Wilkinson, L.; Webb, N.; Weids, A.; Glah, D.; Vrazic, H. A Systematic Literature Review and Network MetaAnalysis Comparing Once-Weekly Semaglutide with Other GLP-1 Receptor Agonists in Patients with Type 2 Diabetes Previously Receiving 1-2 Oral Anti-Diabetic Drugs. Diabetes 2018, 9, 1149-1167. [CrossRef] [PubMed]

22. Witkowski, M.; Wilkinson, L.; Webb, N.; Weids, A.; Glah, D.; Vrazic, H. A Systematic Literature Review and Network MetaAnalysis Comparing Once-Weekly Semaglutide with Other GLP-1 Receptor Agonists in Patients with Type 2 Diabetes Previously Receiving Basal Insulin. Diabetes 2018, 9, 1233-1251. [CrossRef]

23. Sutton, A.; Ades, A.E.; Cooper, N.; Abrams, K. Use of indirect and mixed treatment comparisons for technology assessment. Pharmacoeconomics 2008, 26, 753-767. [CrossRef]

24. Jansen, J.P.; Trikalinos, T.; Cappelleri, J.C.; Daw, J.; Andes, S.; Eldessouki, R.; Salanti, G. Indirect treatment comparison/network meta-analysis study questionnaire to assess relevance and credibility to inform health care decision making: An ISPOR-AMCPNPC Good Practice Task Force report. Value Health 2014, 17, 157-173. [CrossRef]

25. Hutton, B.; Salanti, G.; Caldwell, D.M.; Chaimani, A.; Schmid, C.H.; Cameron, C.; Ioannidis, J.P.; Straus, S.; Thorlund, K.; Jansen, J.P.; et al. The PRISMA extension statement for reporting of systematic reviews incorporating network meta-analyses of health care interventions: Checklist and explanations. Ann. Intern. Med. 2015, 162, 777-784. [CrossRef]

26. Higgins, J.P.; Altman, D.G.; Gøtzsche, P.C.; Jüni, P.; Moher, D.; Oxman, A.D.; Savović, J.; Schulz, K.F.; Weeks, L.; Sterne, J.A. The Cochrane Collaboration's tool for assessing risk of bias in randomised trials. BMJ 2011, 343, d5928. [CrossRef] [PubMed]

27. Cochrane Handbook for Systematic Reviews of Interventions Version 5.0.2 (Updated September 2009). 2009. Available online: http: / / www.cochrane-handbook.org (accessed on 21 July 2018).

28. van Valkenhoef, G. Automating network meta-analysis. Res. Synth. Methods 2012, 3, 285-299. [CrossRef] [PubMed]

29. Wagenmakers, E.J.; Lodewyckx, T.; Iverson, G.J. Bayesian Versus Frequentist Inference. In Bayesian Evaluation of Informative Hypotheses; Hoijtink, H., Klugkist, I., Boelen, P.A., Eds.; Springer: New York, NY, USA, 2008; pp. 181-207.

30. DistillerSR Forest Plot Generator from Evidence Partners. Available online: https://www.evidencepartners.com/resources/ forest-plot-generator/ (accessed on 15 April 2020).

31. Salanti, G.; Ades, A.E.; Ioannidis, J.P. Graphical methods and numerical summaries for presenting results from multiple-treatment meta-analysis: An overview and tutorial. J. Clin. Epidemiol. 2011, 64, 163-171. [CrossRef] [PubMed]

32. Nauck, M.; Frid, A.; Hermansen, K.; Shah, N.S.; Tankova, T.; Mitha, I.H.; Zdravkovic, M.; Düring, M.; Matthews, D.R. Long-term efficacy and safety comparison of liraglutide, glimepiride and placebo, all in combination with metformin in type 2 diabetes: 2-year results from the LEAD-2 study. Diabetes Obes. Metab. 2013, 15, 204-212. [CrossRef] [PubMed]

33. Garber, A.; Henry, R.; Ratner, R.; Garcia-Hernandez, P.A.; Rodriguez-Pattzi, H.; Olvera-Alvarez, I.; Hale, P.M.; Zdravkovic, M.; Bode, B. Liraglutide versus glimepiride monotherapy for type 2 diabetes (LEAD-3 Mono): A randomised, 52-week, phase III, double-blind, parallel-treatment trial. Lancet 2009, 373, 473-481. [CrossRef]

34. Ahrén, B.; Masmiquel, L.; Kumar, H.; Sargin, M.; Karsbøl, J.D.; Jacobsen, S.H.; Chow, F. Efficacy and safety of once-weekly semaglutide versus once-daily sitagliptin as an add-on to metformin, thiazolidinediones, or both, in patients with type 2 diabetes (SUSTAIN 2): A 56-week, double-blind, phase 3a, randomised trial. Lancet Diabetes Endocrinol. 2017, 5, 341-354. [CrossRef]

35. Marso, S.P.; Bain, S.C.; Consoli, A.; Eliaschewitz, F.G.; Jódar, E.; Leiter, L.A.; Lingvay, I.; Rosenstock, J.; Seufert, J.; Warren, M.L.; et al. Semaglutide and Cardiovascular Outcomes in Patients with Type 2 Diabetes. N. Engl. J. Med. 2016, 375, 1834-1844. [CrossRef]

36. Davies, M.J.; Bergenstal, R.; Bode, B.; Kushner, R.F.; Lewin, A.; Skjøth, T.V.; Andreasen, A.H.; Jensen, C.B.; DeFronzo, R.A. Efficacy of Liraglutide for Weight Loss Among Patients With Type 2 Diabetes: The SCALE Diabetes Randomized Clinical Trial. JAMA 2015, 314, 687-699. [CrossRef] [PubMed]

37. Pratley, R. Oral semaglutide versus subcutaneous liraglutide and placebo in type 2 diabetes (PIONEER 4): A randomised, double-blind, phase 3a trial. Lancet 2019, 394, 39-50. [CrossRef]

38. Pratley, R. One year of liraglutide treatment offers sustained and more effective glycaemic control and weight reduction compared with sitagliptin, both in combination with metformin, in patients with type 2 diabetes: A randomised, parallel-group, open-label trial. Int. J. Clin. Pract. 2011, 65, 397-407. [CrossRef]

39. Kaku, K. Safety and efficacy of once-weekly semaglutide vs additional oral antidiabetic drugs in Japanese people with inadequately controlled type 2 diabetes: A randomized trial. Diabetes Obes. Metab. 2018, 20, 1202-1212. [CrossRef]

40. Mishriky, B.M.; Cummings, D.M.; Powell, J.R.; Sewell, K.A.; Tanenberg, R.J. Comparing once-weekly semaglutide to incretinbased therapies in patients with type 2 diabetes: A systematic review and meta-analysis. Diabetes Metab. 2019, 45, 102-109. [CrossRef] [PubMed]

41. Shi, F.H.; Li, H.; Cui, M.; Zhang, Z.L.; Gu, Z.C.; Liu, X.Y. Efficacy and Safety of Once-Weekly Semaglutide for the Treatment of Type 2 Diabetes: A Systematic Review and Meta-Analysis of Randomized Controlled Trials. Front. Pharm. 2018, 9, 576. [CrossRef]

42. Li, X.; Qie, S.; Wang, X.; Zheng, Y.; Liu, Y.; Liu, G. The safety and efficacy of once-weekly glucagon-like peptide-1 receptor agonist semaglutide in patients with type 2 diabetes mellitus: A systemic review and meta-analysis. Endocrine 2018, 62, 535-545. [CrossRef] [PubMed] 
43. Nauck, M.A.; Petrie, J.R.; Sesti, G.; Mannucci, E.; Courrèges, J.P.; Lindegaard, M.L.; Jensen, C.B.; Atkin, S.L. A Phase 2, Randomized, Dose-Finding Study of the Novel Once-Weekly Human GLP-1 Analog, Semaglutide, Compared With Placebo and Open-Label Liraglutide in Patients With Type 2 Diabetes. Diabetes Care 2016, 39, 231-241. [CrossRef]

44. Capehorn, M.S.; Catarig, A.-M.; Furberg, J.K.; Janez, A.; Price, H.C.; Tadayon, S. Efficacy and safety of once-weekly semaglutide $1.0 \mathrm{mg}$ vs once-daily liraglutide $1.2 \mathrm{mg}$ as add-on to $1-3$ oral antidiabetic drugs in subjects with type 2 diabetes (SUSTAIN 10 ) Diabetes Metab. 2020, 46, 100-109. [CrossRef] [PubMed]

45. Webb, N.; Orme, M.; Witkowski, M.; Nakanishi, R.; Langer, J. A network meta-analysis comparing semaglutide once-weekly with other GLP-1 receptor agonists in Japanese patients with type 2 diabetes. Diabetes Ther. 2018, 9, 973-986. [CrossRef] 